\title{
Prácticas de cuidado cultural de mujeres con morbilidad materna extrema
}

\section{Cultural care practices of women with extreme maternal morbidity \\ Práticas de cuidados culturais de mulheres com morte materna extrema}

\author{
Luis Miguel Hoyos Vertel1, Lucy Muñoz de Rodriguez² \\ ${ }^{1}$ Magíster en Enfermería, Universidad Nacional de Colombia, Sede Bogotá, \\ Colombia. Correo electrónico: Imhoyosv@unal.edu.co \\ ${ }^{2}$ Magíster en Enfermería, Profesora Emérita, Universidad Nacional de \\ Colombia, Sede Bogotá. Correo electrónico: lucymdero@yahoo.com \\ Cómo citar este artículo en edición digital: Hoyos Vertel, L. M. \& Muñoz De Rodríguez. L. (2019). \\ Prácticas de cuidado cultural de mujeres con morbilidad materna extrema. Cultura de los Cuidados \\ (Edición digital), 23 (54). Recuperado de http://dx.doi.org/10.14198/cuid.2019.54.24 \\ Correspondencia: Carrera 31A \# 3579 Ciudad: Montería-Colombia \\ Correo electrónico de contacto: Imhoyosv@unal.edu.co \\ Recibido: 10/12/2018; Aceptado: 07/04/2019
}

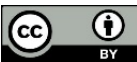

\section{ABSTRACT}

The present article is generated from the results of the research: Beliefs and cultural care practices of women with extreme maternal morbidity (Hoyos \& Muñoz, 2017).

Objective: To describe the cultural care beliefs and practices of women who had extreme maternal morbidity, who were not in control of prenatal care and who were treated at hospital in the city of Medellín, Colombia.

Sample: 17 women with extreme maternal morbidity, not in control of prenatal care.

Method: Qualitative, ethnographic, using ethno-nursing proposed by Leininger and Spradley's ethnographic interview.

Results: 3 general topics and 2 taxonomies were found.

Conclusions: The study concluded that women with extreme maternal morbidity, who do not have prenatal care, have their own ways of caring for themselves and their unborn child when they are sick, because access to maternal health services is poor.

Keywords: Nursing, ethno-nursing, cultural care, health, severe maternal morbidity.

\section{RESUMO}

Este artigo tem sua origen no projeto da pesquisa: Crenças e práticas de cuidados culturais de mulheres com extrema morbidade materna (Hoyos \& Muñoz, 2017).

Objetivo: Descrever as crenças e práticas de atenção cultural de mulheres que tiveram morbidade materna extrema, que não controlavam o pré-natal e que foram atendidas em um hospital da cidade de Medellín, na Colômbia.

Método: Qualitativo, etnográfico, utilizando a etno-enfermagem proposto 
pela entrevista etnográfica de Leininger e Spradley.

Amostra: 17 mulheres com extrema morbidade materna, não controlando o prénatal.

Resultados: Foram encontrados 3 tópicos gerais e 2 taxonomias.

Conclusões: $O$ estudo concluiu que as mulheres com morbidade materna extrema, que não controlam o pré-natal, têm suas próprias formas de cuidar de si mesmas e do nascituro quando estão doentes, porque o acesso aos serviços de saúde materna é fraco.

Palavras chave: Enfermagem, etnoenfermagem, cuidados culturais, saúde, extrema morbidade materna.

\section{RESUMEN}

El presente artículo se genera a partir de los resultados de la investigación: Creencias y prácticas de cuidado cultural de mujeres con morbilidad materna extrema (Hoyos \& Muñoz, 2017).

Objetivo: Describir las creencias y prácticas de cuidado cultural de mujeres que tuvieron morbilidad materna extrema, inasistentes al control prenatal y atendidas en un Hospital de la ciudad de MedellínColombia.

Método: Cualitativo, etnográfico, utilizando la etnoenfermería propuesto por Leininger y la entrevista etnográfica de Spradley.

Muestra: 17 mujeres con morbilidad materna extrema, inasistentes a control prenatal.

Resultados: Se encontró 3 temas generales y 2 taxonomías.

Conclusiones: El estudio concluyó que la mujer con morbilidad materna extrema, inasistente al control prenatal tiene sus propios modos de cuidado a sí misma y al hijo por nacer cuando está enferma, porque el acceso a los servicios en salud materna es deficiente.

Palabras clave: Enfermería, etnoenfermería, cuidado cultural, salud, morbilidad materna extrema.

\section{INTRODUCCIÓN}

La morbilidad materna extrema es "una complicación que ocurre en el embarazo parto y puerperio y que coloca en riesgo la vida de la mujer y del hijo por nacer" (FLASOG, 2007). Muchos casos se presentan por la falta de accesibilidad al servicio de salud materna, lo cual justifica la inasistencia a los programas prenatales en lugares dispersos de Colombia.

Conocer estas creencias y prácticas de cuidado de este grupo poblacional se logró con el referente de la teoría de la universalidad y diversidad del cuidado cultural de Leininger, quien ha definido la enfermería transcultural como "un área principal de la enfermería que se centra en el estudio comparativo y el análisis de las diferentes culturas y subculturas del mundo con respecto a los valores sobre los cuidados, la expresión, las creencias de salud, la enfermedad y prácticas de cuidado, cuyo propósito consiste en concebir un saber científico y humanístico para que proporcione una práctica de cuidados enfermeros específicos para la cultura y/o una práctica de cuidados enfermeros universales de la cultura" (Leininger, 1994). Con base en la teoría de Leininger, se propuso la presente investigación sobre creencias y prácticas de cuidado cultural de gestantes con causas principales de morbilidad materna extrema e inasistentes a control prenatal, con el fin de aportar al cuidado culturalmente congruente en este 
grupo. El concepto de morbilidad materna extrema es versátil de acuerdo con Águila (2012) y la fácil identificación de los casos y la posibilidad de entrevistar las sobrevivientes refuerzan el valor del estudio fortaleciendo la disciplina, al desarrollar planes de cuidado con base a lo planteado por Leininger, lo cual guía al profesional de enfermería en la planificación y ejecución de planes de acciones y políticas en salud materna e infantil con el fin disminuir la mortalidad materna. Diferentes estudios y autores plantean como causas principales de morbilidad materna extrema; hipertensión arterial Jayaratnam (2011), preclamsia Monroy (2012), eclampsia Zanconato (2012), alteración de los factores de coagulación Van den Akker (2013), la diabetes gestacional Adeoye (2013), parto obstruido y prolongado Zanette (2014), hemorragia grave Alvarez (2010), septicemia Lopez (2009) y anemia grave Galvão (2014). Según el Instituto Nacional de Salud de Colombia (INS) para el año 2015 el total acumulado de casos de morbilidad materna extrema a semana epidemiológica 52 fue de 15.067 (INS, 2016). La morbilidad materna extrema, ocurre con mayor frecuencia que la mortalidad materna, lo cual permite un análisis sobre un número mayor de casos y favorece una cuantificación más detallada de los factores de riesgo y determinantes sociales y culturales de salud, que la obtenida al analizar un número relativamente pequeño de muertes maternas (INS, 2014). Los factores determinantes en salud, que influyen en una mujer para desarrollar morbilidad materna extrema pueden estar relacionados con el contexto social, cultural, y entre ellos se destacan el estado de inferioridad económica, educativa, legal o familiar, y los relativos a su estado de salud, así como el acceso y calidad de los servicios de salud para la atención materna y la planificación familiar (INS, 2014). Estudios confirman que una atención de calidad y la asistencia al programa prenatal, es un factor protector para la detección de la morbilidad materna; Jabir (2013) Verma (2013) Rodríguez (2014) Cáceres (2009) Lasso (2012) Munares (2013) Rodríguez (2014) y Choudhury (2011). En el departamento de Antioquia, según la Encuesta Nacional de Demografía y Salud ENDS (Minsalud Colombia, 2015), se reportó asistencia al control prenatal del $94.1 \%$, y de este el $88.4 \%$ de los controles fueron atendidos por Médicos, el 5.4\% Enfermeras (os) Profesionales, $0.6 \%$ parteras, $0.3 \%$ auxiliares de enfermería y el $5.4 \%$ no asistió a control prenatal. Para Medellín, asistencia al control prenatal del 99.8\% de los cuales el $97.3 \%$ los realizó el Médico, y el 2.5\% lo realizó la Enfermera(o) Profesional, y el $0.2 \%$ no recibió controles prenatales. Al realizar la comparación con Antioquia y Medellín, se observó que hay una mayor asistencia al control prenatal, en su capital que en los demás municipios de acuerdo con el Minsalud Colombia (2015). Esta inasistencia al control prenatal, en el caso de Antioquia del 5.4\%, y el índice negativo de $0.2 \%$ de inasistencia en la ciudad de Medellín, impidió detectar tempranamente la morbilidad materna extrema en gestantes que no asisten al control prenatal y hace suponer que estas mujeres tienen sus propios modos de cuidarse desde su propia cultura. Leininger en su teoría de la Universalidad y Diversidad del Cuidado Cultural plantea que en varias de sus observaciones directas y experiencias en personas de diversas culturas, con una variedad de condiciones de salud, encontró que el modo de cuidado humano era importante para la recuperación de una enfermedad, el mantenimiento de la salud y el bienestar y 
que las personas naturalmente tienen sus propias maneras de cuidarse, por lo que retó a las enfermeras a descubrir el cuidado específico y holístico utilizado por las diferentes culturas a través del tiempo y en distintos contextos McFarland (2006). Con los propósitos anteriores, se encontraron investigaciones desde lo cultural en gestantes que han experimentado situaciones de enfermedad, relacionadas con causas principales de morbilidad materna extrema como diabetes y preclamsia, donde se hizo referencia a creencias y prácticas que utilizaron, las mujeres de los estudios, para su cuidado y el de su hijo por nacer. Guerra (2006) encontró que las embarazadas diabéticas consideran la salud como un valor, como algo positivo; Benavente (2008) descubrió el significado que tiene la salud y la enfermedad para un grupo de adolescentes diabéticas, encontrando que tener salud significa estar tranquilas. Álvarez (2012) describió la percepción del cuidado de enfermería en gestantes con preclamsia, encontrando que el $95 \%$ de las gestantes percibió que el personal de enfermería demostró conocimiento y competencias en los cuidados terapéuticos que proporciono frente a la preclamsia. Laza (2012) describió la experiencia de la mujer con preclamsia, encontrando que esta experiencia generó miedo y angustia. Pérez (2011) describió el significado que un grupo de puérperas asignaron a la preclamsia, encontrando que confían en estar sanas y reaccionan ante el diagnóstico con temor al riesgo de morir ella y su hijo. Castiblanco (2014), describió la percepción acerca de la preclamsia, encontrando que las mujeres que sufrieron por primera vez la enfermedad, la percibieron de forma "inesperada y sin avisar"; para las que la habían padecido, la esperaron con resignación y angustia.
Noguera (2014) describió los significados de tener preclamsia para un grupo de gestantes, encontrando que se generan sentimientos como incertidumbre, miedo, nervios y ansiedad, secundario a no saber qué va a pasar con su salud y con la salud del hijo por nacer. Pulido (2014) describió las experiencias vividas por la preclamsia severa, encontrando que la relación con Dios, se convirtió en una fortaleza y compañía en momentos de soledad, incertidumbre y miedo frente al peligro que implicó la enfermedad. Pérez (2009) detectó las necesidades de cuidado percibidas por gestantes con diagnóstico de preclamsia, encontrando que requieren un estricto cuidado en el manejo de cuatro necesidades básicas que son: fisiológicas, seguridad, amor y pertenencia, y de autorrealización. Con lo referido anteriormente, es evidente que los estudios abordaron la clasificación y las causas de la morbilidad materna extrema, la importancia de la calidad de la atención de las gestantes con morbilidad materna extrema, las demoras en la atención y búsqueda de ayudas, la no detección temprana de la morbilidad materna extrema, el control prenatal como un factor protector de la morbilidad materna extrema, la dimensión y presencia de factores culturales como creencias y prácticas que utilizan las gestante con alguna situación de enfermedad relacionada con morbilidad materna extrema. Estudios sobre creencias y prácticas de cuidado desde lo cultural en mujeres que tuvieron causas principales de morbilidad materna extrema y fueron inasistentes a control prenatal, no fueron encontrados, por lo cual surge la necesidad de este estudio.

\section{MÉTODO}

El método utilizado fue cualitativo 
etnográfico desde la Etnoenfermería como método naturalístico y abierto de investigación que permitió estudiar y explicar fenómenos relacionados con la teoría del cuidado cultural (Leininger, 2002).

La técnica utilizada para la recolección de la información fue la entrevista etnográfica y la observación etnográfica de James Spradley, conociendo lo que la gente sabe, piensa y cree (Spradley, 1983), el tipo de preguntas que se hicieron fueron de tipo descriptivo, estructural y de contraste, en promedio de 2 a 3 entrevistas, también se usó las notas de campo con las cuales se logró observar e indagar sobre el contexto y escenario cultural lo cual permitió planificar y orientar nuevas preguntas para entrevistas adicionales con el fin de saturar la información, las entrevistas se grabaron en un dispositivo electrónico casete, posterior a esto se realizó una lectura juiciosa y analítica tratando de entender la información que las participantes compartieron con el investigador para identificar los dominios y posteriormente realizar el análisis componencial. El Análisis de la información se hizo a la par de la recolección, mediante la saturación de la información y no por un número previamente establecido. La investigación evalúo la calidad científica según los criterios de credibilidad, auditabilidad y transferibilidad, (Lincoln, 1985); la credibilidad se aplicó a través de la observación, notas de campo y las entrevistas analizadas y confirmadas por las mujeres del estudio. La confirmabilidad de este estudio se aplicó con la directora de la tesis quien realizó el acompañamiento y seguimiento paso a paso del desarrollo de toda la investigación; a través de revisiones de las entrevistas, notas de campo, análisis de la investigación, sugerencias y envío de material bibliográfico. Para garantizar la validez y confiabilidad, además de la credibilidad y la confirmabilidad, el investigador invirtió el tiempo suficiente para realizar la captación de las participantes y para desplazarse y realizar las entrevistas en el lugar donde se encuentren las gestantes o puérperas, hasta obtener saturación en la información.

El presente proyecto de investigación se realizó bajo los principios de: beneficencia, justicia, autonomía, veracidad y fidelidad, principios importantes en la práctica de enfermería de acuerdo con Tejera (2004). Esta investigación, según la Resolución 8430 de 1983 es de bajo riesgo Minsalud Colombia (1993). Este estudio no realizó ninguna intervención o modificación intencionada de variables fisiológicas, psicológicas o sociales de las participantes. La recolección de la información se hizo cuando se obtuvo la aprobación por parte del Comité de Ética de la Facultad de Enfermería de la Universidad Nacional de Colombia, del Hospital General de Medellín, para utilizar sus instalaciones y captar a las gestantes o puérperas con causas principales de morbilidad materna extrema para incluirlas dentro de la muestra del estudio. En el consentimiento informado se advirtió a las participantes que las entrevistas serán grabadas y posteriormente transcritas textualmente para hacer el análisis respectivo del estudio.

\section{RESULTADOS Y DISCUSIÓN}

\section{Características del contexto}

Fueron 17 Gestantes o puérperas con causas principales de morbilidad materna extrema, inasistentes a control prenatal, atendidas en el Hospital General de Medellín- Colombia. La edad promedio fue de 22 años, el 47\% procedían de Municipios de Antioquia, $41 \%$ de Municipios que 
conforman el área metropolitana del Valle de Aburrá y el 12\% restante fueron referenciadas del departamento del Choco. El 58\% residían en cabeceras municipales, $22 \%$ en rural disperso y el $20 \%$ en centros poblados, la ocupación de las mujeres, $47 \%$ eran amas de casa, $23 \%$ estudiantes, $11 \%$ oficios varios, $9 \%$ operarias de confección, auxiliar de enfermería y vendedoras de pollo 5\% respectivamente. El tipo de régimen en salud, fue subsidiado para $100 \%$ de las mujeres, nivel de escolaridad, el $48 \%$ tenía bachillerato completo, $\quad 17 \%$ bachillerato incompleto, $29 \%$ primaria completa y el $6 \%$ tenía técnica.

\section{Prácticas de cuidado cultural}

Las prácticas de cuidado identificadas en las mujeres con morbilidad materna extrema evidencian prácticas de cuidado desde lo profesional (ético) y desde lo genérico (émico). Las primeras corresponden a las orientadas por profesionales de la salud, las genéricas provenientes de su conocimiento ancestral. Al realizar el análisis de los datos obtenidos surgen los siguientes temas de la investigación, los cuales cada uno de estos es presentado, en tabla con sus respectivos dominios y una taxonomía. Las demás taxonomías son descritas en texto de la respectiva descripción del dominio.

\section{Prácticas de cuidado desde lo profesional}

Las prácticas de cuidado desde lo profesional que realizan las mujeres con morbilidad materna extrema, están basadas en las orientaciones del equipo de profesionales de la salud y en algunos casos particulares han sido aprendidas por medios de comunicación masiva como lo es la internet y las aplicaciones móviles. "aprendí a cuidarme con la aplicación baby center" (P2E1), "en la aplicación aprendí de la alimentación" (P1E1). Las mujeres con morbilidad materna extrema suelen cuidarse a través de prácticas de cuidado como son la alimentación; consumiendo una dieta rica en proteínas, baja en lípidos, carbohidratos y sodio, "comer de todo, carne pollo, frutas, verduras, harinas, arroz, pescado" (P1E1, P2E1), muchas dicen que esta práctica en el caso de las mujeres con preclamsia la realizan para evitar que su tensión arterial se eleve, tengan edema, al igual que su glicemia en el caso de las mujeres con diabetes gestacional, "evitar comer azucares, $y$ cosas dulces" (P9E1, P6E1), "el médico me dijo que si no disminuía el dulce quedare con azúcar" (P12E1), "no comer salado por el edema" (P13E1, P16E1, P11E1), también ellas realizan ejercicio físico y cardiovascular, "trotar por 20 a 45 minutos" (P2E1, P3E2), "si trotas tu hijo nace bien y te va bien cuando pares, te pones feliz" (P4E1), puesto que esto las ayuda a mejorar la circulación sanguínea su sistema cardiovascular y así mejorar la irrigación y perfusión tisular a su hijo, también descansan, se relajan y evitan realizar esfuerzo físico, "dormir cómoda, descansar constantemente y dormir plácidamente" (P8E1, P3E2), "no trasnocharse, dormir sin ruidos" (P15E1), "si duermes bien él bebé crece más rápido y sale sanito" (P9E2, P6E1), "ir al yoga te ayuda a estar relajada" P5E1, "moverse poquitico, o no moverte para que no se te venga él bebe" (P5E1, P6E1), con el fin de prevenir abortos espontáneos y partos pretérmino, otras prácticas de cuidado que realizan es el cuidado así misma y al hijo por nacer asistiendo a los controles prenatales, "me han enseñado como cuidarme" (P3E2, P7E1, P8E1, P9E1), y consultando por urgencias cuando se presenten signos de alarma "cuando tengo signos de alarma ir para la urgencia enseguida" (P12E1, P6E2), "si me pongo mal me voy para urgencia me dijo la jefe" (P4E1, P5E1), "Ir para la urgencia cuando tenga 
dolores de cabeza, mareo" (P4E1, P5E1),"si tengo vómito, si estoy amarilla" (P13E1, P15E1)"si se me sale el líquido, sangrado, si no se mueve" (P12E1, P6E2), como son dolores de cabeza, mareo, vomito, si presentan ictericia, edema, salida de líquidos por vía vaginal y/ sangrado, si no se mueve el feto y visión borrosa.

A pesar de que este grupo de mujeres con morbilidad materna extrema no asistió puntualmente al programa detección temprana de alteraciones del embarazo (control prenatal), de acuerdo con la norma 412 de 2000 Minsalud Colombia (2000), ellas han aprendido del grupo de profesionales de la salud a cuidarse y a mantener hábitos y estilos de vida que puedan contribuir a mejorar su condición de salud y calidad de vida y la de su hijo por nacer.

Lo anterior se puede concluir que estas prácticas de cuidado son congruentes con las orientaciones que recomiendan organismos de salud.

Algunas investigaciones confirman los anteriores argumentos y razones del cuidado de la mujer; Benavente (2008) encontró que las mujeres diabéticas restringen el consumo de algunos alimentos para mantenerse sanas. Jabir (2013) Verma (2013) y Rodríguez (2014) detectaron que la mujer debe identificar tempranamente los signos de alarma, con el fin de tener una atención de calidad. Lira (2014) encontró que las gestantes de la etnia otomí evitaban cargar cosas pesadas. Muñoz (2015) encontró que la mujer mantiene prácticas de cuidado de forma regular en la alimentación.

\section{Prácticas de cuidado desde lo genérico}

Las prácticas de cuidado genérico que realizan las mujeres con morbilidad materna extrema están basadas en un conocimiento folclórico y cultural, ellas practican esos cuidados basados en el saber de su experiencia personal y la de sus parientes cercanos las cuales han sido transmitidos de generación en generación. Este saber está basado en prácticas culturales relacionadas con el uso de hierbas, "tomar baño de hojas de eucalipto, canela, manzanilla, lulo, hojas de brevo, sentada en una silla y recibir ese vapor" (P1E1, P14E1, P8E1, P17E1, P16E1), "tomar agua de timorreal, lulo, y hojas de brevo con panela" (P3E1, P2E2, P3E1), "tomar aceite de recino para el dolor de parto" (P4E1, P5E1) "echarse eucalipto en la barriga" (P9E2), evitar movimientos bruscos, "moverse poquitico, o no moverte para que no se te venga él bebe" (P5E1, P6E1) "permanecer quieta" (P7E2, P17E1), "no hacer mayor cosa" (P2E2), estar tranquila "estar siempre relajada y no coger rabietas por riesgo al aborto" (P15E1), "el yoga me pone tranquila a mí y al bebe" (P1E1), "ir a control prenatal me calma" (P2E2, P8E2), "que te atienda el ginecólogo te mantiene tranquila" (P7E1, P9E1, P15E1), "me gusta que me atienda la jefe, (enfermera(o) profesional) me deja más tranquila" (P14E1), protegerse de cambios térmicos, "no serenarse" (P8E1, P9E1), "no acercarme a sitios donde pase el frio" (P7E1, P12E1), "no salir si está lloviendo" (P7E1, P10E2), "no sentarse en el piso frio" P5E1, "salir con chaqueta" (P17E1, P13E1), "no exponerme a esos frio, porque sale flaquito él bebe" (P9E1), "uso blusas largas, igual allá hace calor, pero el frio de la noche" (P14E1), precauciones antes del parto, "él bebe se salva después de los 7 meses" (P8E1), "no comprarle ropa al bebe" (P8E1), "lo hago por supersticiones de mi familia" (P8E1), "se le compra la ropita después de los 8 meses (P8E1), la higiene, bañarse para evitar infecciones y trasmitirlas al hijo" (P8E1, P11E1) "bañarse las partes bien (genitales)" (P9E1), los cambios de posición ante la presencia de edema, la mujer con morbilidad materna extrema cuida de ella misma y del hijo por nacer en ausencia de los 
controles prenatales, cuando evidencia edema realiza prácticas como elevación de miembros inferiores; "levanto los pies para que se me quite la hinchazón" (P9E2, P16E1, P14E1), "colocar piedras en los pieseros para bajar la cabecera de la cama" (P8E1), "acostarse del lado izquierdo" (P6E1, P5E1), la restricción en el consumo de agua y líquidos, disminuyen el consumo de líquido como practica para evitar que el edema aumente; "no consumir agua para que se me disminuya la hinchazón" (P16E1, P6E1, P14E1) y la búsqueda de ayuda ante problemas de salud "la enfermera del pueblo me toma la presión y estaba alta" (P6E1), "consulte en la urgencia por la presión" (P12E1, P15E1), "llamo a una enfermera cuando tengo dolor de cabeza" (P4E1, P1E1), "me Marie y fui a urgencias enseguida" (P3E2), "sentí unos pálpitos en el corazón y fui al hospital del pueblo" (P13E1).

Estas prácticas son valiosas tanto para las mujeres con morbilidad materna extrema como para el sistema sanitario y de salud, ya que por medio de estas cuidan su embarazo, a su hijo por nacer, ayudan en el proceso de parto, disminuyendo los problemas de salud reales y potenciales que puedan presentar, lo mismo que el cuidado frente a situaciones adversas como lo es la enfermedad cuando se está embarazada. Es así como las prácticas de cuidado genérico que realizan estas mujeres consigo mismo y con el hijo por nacer ejercen una importancia en el mejoramiento de su condición de salud y de vida, puesto que este saber folclórico $y$ cultural le brinda herramientas para cuidarse, para cambiar su estilo de vida y garantizar un embarazo sano a pesar de su condición clínica. Sin embargo, la confianza que ellas tienen en estas prácticas puede convertirse en un factor de riesgo para la morbilidad materna puesto que reemplaza la consulta del servicio de salud, por sus prácticas de cuidado folclóricas. Parra (2011) encontró que las gestantes en su mayoría tienen buena práctica de cuidado de limpieza e higiene de los genitales y del cuerpo; lo cual contribuye a disminuir las infecciones del tracto genitourinario, factor de riesgo para amenazas de aborto y parto prematuros, una práctica de cuidado similar que usan las gestantes de la presente investigación. Choudhury (2011), encontró que las mujeres consideran el embarazo como un evento normal, a menos que surjan complicaciones y en ese momento buscan ayuda profesional.

\section{CONCLUSIONES}

Las mujeres con morbilidad materna extrema suelen cuidarse a través de prácticas de cuidado como la alimentación, evitando el consumo de algunos alimentos que ella considera perjudiciales para su estado, también ellas realizan ejercicio físico mejorando la irrigación y perfusión hística tisular a su hijo, también descansan, se relajan y evitan realizar esfuerzo físico con el fin de prevenir abortos espontáneos y partos pretérmino. Muchas de estas prácticas son propias y a su vez recomendadas por profesionales de la salud. Las prácticas de cuidado genérico están basadas en un conocimiento folclórico y cultural, basados en su experiencia personal y la de sus parientes cercanos las cuales han sido transmitidos de generación en generación, entre ellas uso de hierbas, estar tranquila, no exponerse al frio, precauciones ante el parto, cambiar posición ante la presencia de edema, restringir el consumo de agua, líquidos y sodio, por medio de estas cuidan su embarazo, a su hijo por nacer. El uso de estas prácticas evidencia que muchas mujeres no consultan porque prefieren cuidarse en su hogar, a pesar de estar enfermas. Es imperativo tener en cuenta el 
conocimiento genérico (émico) de las mujeres con morbilidad materna extrema durante la atención en el programa detección temprana de las alteraciones del embarazo (control prenatal) y durante su estancia hospitalaria, educándolas y preservando y manteniendo el cuidado genérico (émic) adecuado y benéfico, negociando o acomodando las susceptibles de mejorar y reestructurando las prácticas que pueden ser perjudiciales para ellas.

\section{REFERENCIAS BIBLIOGRÁFICAS}

- Adeoye, I. y Onayade, A. (2013). Incidence, determinants and perinatal outcomes of near miss maternal morbidity in Ile-Ife Nigeria: a prospective case control study. BMC pregnancy and childbirth; 13(1), $93 . \quad$ Recuperado de https://bmcpregnancychildbirth.biomedcentral.com/ articles/10.1186/1471-2393-13-93.

- Águila, S. (2012). Una estrategia para la disminución de la mortalidad materna. Revista Cubana de Obstetricia y Ginecología, 38(2), 281-289. Recuperado de http://ref.scielo.org/ysjd64.

- Álvarez M., Salvador, S. y González, G. (2010). Caracterización de la morbilidad materna extremadamente grave. Revista Cubana de Higiene y Epidemiología; 48(1), 310-320. Recuperado de http://ref.scielo.org/rv5695.

- Álvarez, M. López, R. y Carbonell, I. (2012). Características epidemiológicas de la morbilidad materna extremadamente grave en tres provincias de Cuba, 2009. Revista Cubana de Higiene y Epidemiología; 50(1), 286-299. Recuperado de http://ref.scielo.org/h32pmw.

- Fayad, Y. y López, R. (2009). Materna crítica durante el período 2004-2008. Revista Cubana de Obstetricia y Ginecología; 35(4), 12-19. Recuperado de http://ref.scielo.org/8tmvtg.

- Álvarez, L. y Espitia, S. (2012). Percepción del cuidado de enfermería en gestantes diagnosticadas con preclamsia en una institución de cuarto nivel (Tesis de pregrado), Bogotá: Pontificia Universidad Javeriana. Recuperado de https://repository.javeriana.edu.co/handle/10554/137 $\underline{40}$.

- Benavente, M. Guerra, A. y Mendoza, N. (2008). Significado de la salud-enfermedad desde la perspectiva de las adolescentes diabéticas embarazadas. Enfermería, M., Reproductiva, S., Biblioteca Lascasas; 4(5). Recuperado de http://www.index-

f.com/lascasas/documentos/lc0381.pdf.

- Cáceres, F. (2009). El control prenatal: una reflexión urgente. Revista colombiana de obstetricia y ginecología; 60(2), 165-170. Recuperado de: https://revista.fecolsog.org/index.php/rcog/article/vi ewFile/342/358.

- Choudhury, N. y Ahmed, S. (2011). Maternal care practices among the ultra poor households in rural Bangladesh: a qualitative exploratory study. $B M C$ pregnancy and childbirth; 11(1): 15. Recuperado de https://bmcpregnancychildbirth.biomedcentral.com/ articles/10.1186/1471-2393-11-15.

- FLASOG, (2007). Federación Latinoamericana de sociedades de obstetricia y ginecología. Santa Cruz de la Sierra Bolivia. Recuperado de www.flasog.net.

- Galvão, L. y Alvim, F. (2014). The prevalence of severe maternal morbidity and near miss and associated factors in Sergipe, Northeast Brazil. BMC pregnancy and childbirth; 14(1), 25. Recuperado de https://www.ncbi.nlm.nih.gov/pubmed/24433516.

- Guerra, C. y Vásquez, M. (2006). El cuidado de sí de la embarazada diabética como una vía para asegurar un hijo sano. Texto y Contexto Enfermagem; 15(1), 7481. Recuperado de: http://ref.scielo.org/nhp8hc.

- Jabir, M. y Abdul-Salam, I. (2013). Maternal near miss and quality of maternal health care in Baghdad, Iraq. BMC pregnancy and childbirth; 13(1), 11. Recuperado de

https://bmcpregnancychildbirth.biomedcentral.com/ articles/10.1186/1471-2393-13-11.

- Jayaratnam, S. De Costa, C. y Howat, P. (2011). Developing an assessment tool for maternal morbidity 'near-miss'-A prospective study in a large Australian regional hospital. Australian and New Zealand Journal of Obstetrics and Gynaecology; 51(5), 421-425. Recuperado de https://www.ncbi.nlm.nih.gov/pubmed/21806590.

- Lasso, P. (2012). Atención prenatal: ¿tensiones o rutas de posibilidad entre la cultura y el sistema de salud? Pensamiento psicológico; 10(2), 123-133. Recuperado de http://ref.scielo.org/vmz264.

- Laza, C. y Castiblanco, N. (2014). Peligro, muerte y secuelas: percepción de la preclamsia severa por quienes la han vivido. Enfermería Global; 13(33), 481492. Recuperado de http://www.redalyc.org/articulo.oa?id=365834853022

- Laza, C. y Pulido, G. (2014). La vivencia de la preclamsia: una dura travesía para la cual no se está preparada. Revista Salud UIS; 46(2), 1-7. Recuperado de

http://revistas.uis.edu.co/index.php/revistasaluduis/ 
article/view/4365/6115.

- Laza, C. y Pulido, G. (2012). Cuando la preclamsia irrumpe inesperadamente en el embarazo: dolor, miedo y fe en Dios. Index de Enfermería; 21(4), 234-238. Recuperado de http://ref.scielo.org/z8s9mj.

- Leininger, M., y McFarland, M. (2006). Culture care diversity and universality: A worldwide nursing theory. Boston, Estados Unidos: Jones y Bartlett Learning.

- Leninger, M. (1994). Transcultural nursing. Nursing Education: An International Perspective-. Johanesburgo, South Africa: Hilla Brink Juta and Company.

- Leininger, M. McFarland, M. (2002). Transcultural nursing concepts, theories, research y practice. Washington, Estados Unidos: National League Nursing.

- Lincoln, Y. y Guba, E. (1985). Naturalistic inquiry. Beverly Hills: Sage.

- Lira, B. y Álvarez, A. (2014). Prácticas culturales de cuidado de las mujeres otomíes durante su embarazo. Ene; 8(1). Recuperado de http://ref.scielo.org/smhkxj.

- Ministerio De Salud y Protección Social Colombia. (2016). Encuesta Nacional de Demografía y Salud Profamilia, 2015. Recuperado de https://profamilia.org.co/investigaciones/ends/.

- Ministerio De Salud y Protección Social. (2016). Instituto Nacional De Salud. Boletín epidemiológico Nacional. Semana Epidemiológica 52, 2016. Recuperado de http://www.ins.gov.co/boletinepidemiologico/Boletn $\% 20$ Epidemiolgico/2016\%20B olet $\%$ C3\%ADn $\% 20$ epidemiol $\%$ C3\%B3gico $\% 20$ sema na\%2052\%20-.pdf.

- Ministerio De Salud y Protección Social. (2014). Instituto Nacional De Salud. Vigilancia y Análisis del Riesgo en Salud Publica. Protocolo de Vigilancia en Salud Pública Mortalidad Materna. Recuperado de http://www.ins.gov.co/lineas-deaccion/Subdireccion-

Vigilancia/sivigila/Protocolos\%20SIVIGILA/PRO $\% 2$ 0Morbilidad\%20Materna\%20Extrema.pdf.

- Ministerio de Salud Colombia. Resolución, N. 8430. (1993). Por la cual se establecen las normas científicas, técnicas y administrativas para la investigación en salud. Recuperado

de https://www.minsalud.gov.co/sites/rid/Lists/Bibliote caDigital/RIDE/DE/DIJ/RESOLUCION-8430-DE1993.PDF.

- Monroy, A. Becerril, G. y Vargas, A. (2012). Morbilidad materna extrema (near miss) muertes maternas. Arch Inv Mat Inf; 4(1), 146-53.

- Munares, G. (2013). Factores asociados al abandono al control prenatal en un hospital del Ministerio de Salud Perú. Revista Peruana de Epidemiología; 1(1), 1-8.
- Muñoz, M. y Pardo, M. (2015). Significado de las prácticas de cuidado cultural en gestantes adolescentes de Barranquilla. Aquichan; 16(1), 115126. Recuperado de http://ref.scielo.org/xz6qkj.

- Noguera, N. y Muñoz, L. (2014). Significados que las gestantes hospitalizadas le atribuyen a la experiencia de tener preclamsia. Investigación en Enfermería: Imagen y Desarrollo; 16(1), 27-48.

- Parra, R. y Medina, B. (2011). Prácticas de cuidado de la gestante con ella misma y con su hijo por nacer. Revistas Salud UIS; 43(1), 27-32.

- Pérez, B. y Muñoz, L. (2011). Significado que las puérperas asignan a la experiencia de una gestación con preclamsia (Tesis de maestría). Bogotá: Universidad Nacional de Colombia. Recuperado de http://www.bdigital.unal.edu.co/5415/1/belialuisape rezherrera.2011.pdf.

- Pérez, M. y Prieto, O. (2009). Preclamsia leve: cuidados en casa. Repertorio de Medicina y Cirugía; 18(4), 2-18.

- Rodríguez, F. y Jiménez, W. (2014). Efecto de las barreras de acceso sobre la asistencia a citas de programa de control prenatal y desenlaces perinatales*. Gerencia y Políticas de Salud; 13(27), 1-9.

- Rodríguez, P. y Zapata, V. (2014). Causas de demora en la atención de pacientes con complicaciones obstétricas ¿qué es necesario atender? Ginecol Obstet Mex 82, 647-658.

- Tejera, G. y Pérez, C. (2004). Enfermería familiar y social. La Habana: Editorial Ciencias Médicas.

- Van den Akker, T. y Beltman, J. (2013). The WHO maternal near miss approach: consequences at Malawian District level. PLoS One; 8(1), 54-85. Recuperado de: http://journals.plos.org/plosone/article?id=10.1371/jo urnal.pone.0054805.

- Verma, S. Rai, L. Kumar, P. y Pai, M. (2013). “Near Miss" Obstetric Events and Maternal Deaths in a Tertiary Care Hospital: An Audit. Journal of pregnancy. 5(2), 3-5 Recuperado de http://dx.doi.org/10.1155/2013/393758.

- Zanconato, G. y Cavaliere, E. (2012). Severe maternal morbidity in a tertiary care centre of northern Italy: a 5-year review. The Journal of Maternal-Fetal y Neonatal Medicine; 25(7), 1025-1028.

- Zanette, E. y Parpinelli, M. (2014). Maternal near miss and death among women with severe hypertensive disorders: a Brazilian multicenter surveillance study. Reprod Healt; 11(1), 4. Recuperado de https://www.ncbi.nlm.nih.gov/pubmed/24428879. 